\title{
CRESCIMENTO, ABSORÇÃO E EXPORTAÇÃO DE NUTRIENTES POR UMA CULTURA DE URUCU
}

\author{
H.P. HAAG'; D.L. ROSALEN²; S. SIMÃO ${ }^{3}$; K. MINAMI ${ }^{4}$
}

RESUMO: Sementes de urucu (Bixa orellana L.) var. Peruana foram postas para germinar num substrato constituido de duas partes de terra para uma de matéria orgânica, onde foram adicionados $1,5 \mathrm{~g}$ de superfosfato triplo por litro de substrato. Após 115 dias as plantas foram transplantadas para um Latossolo Vermelho Escuro Orto série "Luiz de Queiroz" de alta fertilidade natural. O espaçamento foi de $5 \mathrm{~m} \times 5 \mathrm{~m}$. Aos 201 dias foi feita uma adubação em cobertura com $5 \mathrm{~g}$ de $\mathrm{KCl}+10 \mathrm{~g}$ de uréia por planta. Plantas foram coletadas a partir dos 115 dias com intervalos de 60 dias até a produção aos 507 dias. As plantas em número de quatro por amostragem foram subdivididas em folhas, ramos, caule e orgãos reprodutivos. Aos 507 dias foram coletados frutos que foram subdivididos em casca e sementes. Os resultados mostraram que o urucu cresce lentamente até aos $\mathbf{2 6 7}$ dias intensificando o seu crescimento até os $\mathbf{4 4 7}$ dias quando ocorre uma paralização. A extração de macro e micronutrientes por uma cultura de 400 plantas $/$ ha aos 507 dias foi de: $N-25,7 \mathrm{~kg}, P$ - $4,1 \mathrm{~kg}, \mathrm{~K}$ $16,7 \mathrm{~kg}, \mathrm{Ca}-16,4 \mathrm{~kg}, \mathrm{Mg}-4,4 \mathrm{~kg}, \mathrm{~S}-2,5 \mathrm{~kg}, \mathrm{~B}-56,4 \mathrm{~g}, \mathrm{Cu}-16,2 \mathrm{~g}, \mathrm{Fe}-240-130,3 \mathrm{~g}$ e $\mathrm{Zn}-50,7 \mathrm{~g}$.

Descritores: Bixa orellana, crescimento, extração, macronutrientes, micronutrientes.

\section{GROWTH, NUTRIENT ABSORPTION AND NUTRIENT EXPORT BY ANATO PLANTS}

ABSTRACT: Anato plant seeds (Bixa orellana var. Peruana) were germinated in a soil + organic matter (2:1) substrate that received triple superphosphate (1.5 g/l). After 115 days, plants were transplanted to a high fertility Dark Red Latosol. Spacing was $5 \mathrm{~m} \times 5 \mathrm{~m}$ a top dressing with $5 \mathrm{~g}$ of $\mathrm{KCl}$ and $10 \mathrm{~g}$ urea per plant was applied at the 201 day. Starting on day 115 plants (4 per sampling) were collected at 60 day intervals until the 507 day. Samples were subdivided in leaves, branches, stem and reproductive parts. At the 507 day fruits were taken and divided into seeds and hulls. Anato plants grew slowly until the $\mathbf{2 6 7}$ day and improved growth rate from then on to the $\mathbf{4 4 7}$ day, when growth almost stopped. The macro and micronutrient extraction by a $\mathbf{4 0 0}$ plant per hectare crop at the 507 day were: $\mathrm{N}-25.7 \mathrm{~kg}, \mathrm{P}-4.1 \mathrm{~kg}, \mathrm{~K}-16.7 \mathrm{~kg}, \mathrm{Ca}-16.4 \mathrm{~kg}, \mathrm{Mg}-4.4 \mathrm{~kg}, \mathrm{~S}$ $-2.5 \mathrm{~kg}, \mathrm{~B}-56.4 \mathrm{~g}, \mathrm{Cu}-16.2 \mathrm{~g}, \mathrm{Fe}-240.1 \mathrm{~g}, \mathrm{Mn}-130.3 \mathrm{~g}$ and $\mathrm{Zn}-50.7 \mathrm{~g}$.

Key Words: Bixa orellana, growth, nutrient absorption, nutrient exportation, macronutrients, micronutrients, anato.

\section{INTRODUÇÃO}

O urucu pertence a família botânica Bixaceae, sendo uma planta perene, originária da América Tropical e Indias Orientais, constituindo-se num arbusto de rápido crescimento podendo atingir de 2 a 5 metros de altura, JOLY (1977). Produz frutos ovóides, tipo cápsula, chamadas de "cachopas", os quais comportam em seu interior sementes envoltas por um arilo vermelho, que the dão a cor característica. Este arilo contém os pigmentos usados na indústria como corantes - a oretina e a bixina. Segundo o Anuário
Estatístico do Brasil (1990) o Brasil produziu em $1989,1.209$ t sendo os maiores produtores o Estado da Paraíba (458t) e o Estado de Minas Gerais com 450t de sementes. Quanto às exigências nutricionais a área 6 praticamente inexplorada. Assim CARVALHO (1986) procedendo a um levantamento bibliográfico encontrou 449 trabalhos abordando diversos aspectos da cultura, sem nenhuma referência bibliográfica sobre nutrição mineral.

Recentemente, ROSALEM et al. (1991) procedendo a um levantamento bibliográfico a cerca da

\footnotetext{
1 Professor Titular, Departamento de Química, ESALQ/USP, Piracicaba,SP.

2 Engenheiro Agrônomo, ESALQ/USP, Piracicaba,SP.

${ }^{3}$ Professor Catedrático (Aposentado), Departamento de Horticultura, ESALQ/USP, Piracicaba,SP.

4 Professor Titular, Departamento de Horticultura, ESALQ/USP, Piracicaba,SP.
} 
cultura encontraram somente o trabalho de HAAG et al. (1988) sobre deficiências nutricionais de $\mathbf{N}, \mathbf{P}, \mathrm{K}, \mathrm{Ca}$, $\mathrm{Mg}$, $\mathrm{S}$ e $\mathrm{B}$, conduzido sob condições controladas em casa de vegetação.

$O$ presente trabalho tem como finalidade:

- apresentar o desenvolvimento vegetativo da cultura em função da matéria seca produzida;

- fornecer as concentrações e as extrações de $\mathrm{N}$, P, K, Ca, Mg, S, B, Cu, Fe, Mn e Zn em diferentes idades;

- quantificar a exportação destes nutrientes pela cultura por ocasião da colheita.

\section{MATERIAIS E METODOS}

Sementes de Bixa orellana $L$. var. Peruana foram postas a germinar em sacos de plástico preto contendo como substrato duas partes de terra para uma de matéria orgânica, adicionados $1,5 \mathrm{~g}$ de superfosfato triplo por litro de substrato. Após 115 dias as plantas foram transplantadas para o solo Latossolo Vermelho Escuro Orto, série "Luiz de Queiroz" de elevada fertilidade natural, como se observa na Tabela 1 , no espaçamento de $5 \times 5 \mathrm{~m}$. Os dados metereológicos acham-se na Tabela 2. Aos 201 dias foi feita uma adubação de cobertura com $5 \mathrm{~kg} \cdot \mathrm{ha}^{-1}$ de $\mathrm{KCl}+10 \mathrm{~g}$ de uréia por planta. Plantas inteiras foram coletadas pelo corte rente ao solo a partir dos 115 dias de campo com intervalos de 60 dias até a produção aos 507 dias. As plantas em número de quatro por amostragem foram subdivididas em folhas, ramos + caules e órgãos reprodutivos (botões florais, somente na coleta aos 447 dias e aos 507 dias foram coletados frutos que foram subdivididos em casca e sementes).

As diferentes partes das plantas foram secas em estufa à $80^{\circ} \mathrm{C}$ e determinado o peso da matéria seca e as concentrações de $\mathrm{N}, \mathrm{P}, \mathrm{K}, \mathrm{Ca}, \mathrm{Mg}, \mathrm{S}, \mathrm{B}, \mathrm{Cu}, \mathrm{Fe}, \mathrm{Mn}$ e $\mathrm{Zn}$, de acordo com a metodologia descrita em SARRUGE \& HAAG (1974).

\section{RESULTADOS E DISCUSSÃO}

\section{Crescimento:}

Pela observação da Tabela 3 nota-se que o crescimento em altura é relativamente lento nos primeiros 267 dias após o plantio acelerando-se após esta data, atingindo o crescimento mais intenso entre 327 e 387 dias de idade. Após os 447 dias há uma queda no crescimento devido a caducidade precoce das folhas (BALIANE, 1982). Este fenômeno pode ser observado no peso da matéria seca das folhas. Os ramos e caule aumentam de peso em matéria seca até aos 447 dias como era de se esperar.

\section{Concentração e extração de macronutrientes:}

A Tabela 4 apresenta a concentração dos macronutrientes nas diversas partes da planta e o correspondente a 400 plantas/ha. Observa-se que nas folhas e nos ramos a concentração percentual diminue levemente $\mathrm{em} \mathrm{N \% ,K \%}$ e S\% com o aumento da idade das plantas sendo que para o $\mathrm{P} \%$ e $\mathrm{Mg} \%$ ocorre pouca variação na concentração. A concentração de $\mathrm{Ca} \%$ aumenta levemente com a idade da planta. $O$ mesmo fenômeno ocorre nos ramos e tronco.

A extração total aumenta como aumento da idade da planta como era de se esperar. Parece que o ponto crítico na extração dos macronutrientes ocorre em torno dos 267-327 dias, ocasião em que os elementos deverão estar em maior disponibilidade no solo, afim que não ocorram limitações no crescimento e produção de sementes. A produção de sementes exporta através da colheita: $20 \% \mathrm{~N}, 31 \% \mathrm{P}, 40 \% \mathrm{~K}, 6 \% \mathrm{Ca}, 16 \% \mathrm{Mg}$ e $19 \% \mathrm{~S}$ do total de nutrientes presentes na planta aos 447 dias de idade. A ordem decrescente da extração total dos macronutrientes aos 507 dias foi: $\mathrm{N}(25,7 \mathrm{~kg})$, $\mathrm{K}(16,74 \mathrm{~kg}), \mathrm{Ca}(16,45 \mathrm{~kg}), \mathrm{Mg}(4,43 \mathrm{~kg}), \mathrm{S}(2,52 \mathrm{~kg})$ e $P(1,18 \mathrm{~kg})$.

\section{Concentração e extração de micronutrientes:}

Pelo exame da Tabela 5 observa-se que as concentrações de $\mathrm{B}, \mathrm{Cu}, \mathrm{Fe}, \mathrm{Mn}$ e $\mathrm{Zn}$ variam durante o desenvolvimento da planta. A extração total em g/ha dos micronutrientes aumenta com a idade da planta especialmente no período de 267 a 327 dias de idade da cultura. A extração total dos micronutrientes por ha aos 507 dias foi de: $\mathrm{B}-56,4 \mathrm{~g}, \mathrm{Cu}-16,1 \mathrm{~g}, \mathrm{Fe}-240,2 \mathrm{~g}$, $\mathrm{Mn}-130,4 \mathrm{~g}$ e $\mathrm{Zn}-50,7 \mathrm{~g}$. Percentualmente em função do total de micronutrientes na cultura a parte exportada (sementes + casca) contribuiu com: B - 22,8\%, Cu $84,5 \%, \mathrm{Fe}-71,90 \%, \mathrm{Mn}-22,1 \%$ e $\mathrm{Zn}-28,2 \%$.

\section{CONCLUSÕES}

- O crescimento do urucu é lento até aos 267 dias de idade sendo que após os 447 dias há uma estabilização.

- Os limites das concentrações percentuais dos macronutrientes nas folhas são: $\mathbf{N}-1,95 \%-3,37 \% ; \mathrm{P}$ $0,20 \%-0,33 \% ; \mathrm{K}-0,89 \%-2,25 \% ; \mathrm{Ca}-0,80 \%$ $1,96 \% ; \mathrm{Mg}-0,38 \%-0,43 \% ; \mathrm{S}-0,15 \%-0,28 \%$. 
Tabela 1 - Análise do solo da área experimental e interpretações.

\begin{tabular}{|c|c|c|c|c|c|c|c|c|c|}
\hline P resina & M.O. & pH & $\mathbf{K}$ & $\mathrm{Ca}$ & $\mathbf{M g}$ & $\mathrm{H}+\mathrm{Al}$ & $\mathbf{S}$ & CTC & V\% \\
\hline ppm & $\%$ & $\left((\mathrm{Cacl})_{2}\right.$ & \multicolumn{7}{|c|}{$\mathrm{meq} / 100 \mathrm{~cm}^{3}$} \\
\hline 112,4 & 3,20 & 5.45 & 0.44 & 4,32 & 0,72 & 3,25 & 5,50 & 8,70 & 62,80 \\
\hline $\begin{array}{l}\text { Muito } \\
\text { alto }\end{array}$ & Alto & Médio & Alto & Alto & Médio & Baixo & Alto & Médio & Médio \\
\hline
\end{tabular}

Tabela 2 - Dados climatológicos nas épocas amostradas (*).

\begin{tabular}{|c|c|c|c|c|c|c|c|c|}
\hline \multirow{2}{*}{$\begin{array}{l}\text { Idade } \\
\text { (dias) }\end{array}$} & \multirow[b]{2}{*}{ Data } & \multirow{2}{*}{$\begin{array}{l}\text { Radiação } \\
\text { Global } \\
\left.\text { (cal/ } \mathrm{cm}^{2} \mathrm{dia}\right)\end{array}$} & \multirow{2}{*}{$\begin{array}{l}\text { Insolação } \\
\text { (h/dia) }\end{array}$} & \multirow{2}{*}{$\begin{array}{l}\text { Precipi- } \\
\text { tação } \\
(\mathrm{mm})\end{array}$} & \multirow{2}{*}{$\begin{array}{l}\text { Umidade } \\
\text { Relativa } \\
(\%)\end{array}$} & \multicolumn{3}{|c|}{ Temperatura $\left({ }^{\circ} \mathrm{C}\right)$} \\
\hline & & & & & & Máxima & Mínima & Média \\
\hline 115 & 4.abr. 88 & 300 & 5.9 & 140.00 & 75. & 28.6 & 17.2 & 22.00 \\
\hline 176 & 4.jun. 88 & 267 & 7.2 & 26.80 & 65. & 23.6 & 9.2 & 16.40 \\
\hline 206 & 4.ago. 88 & 358 & 9.5 & 0.00 & 52. & 28.6 & 10.0 & 19.30 \\
\hline 267 & 4.out. 88 & 341 & 6.0 & 196.90 & 63. & 28.6 & 15.3 & 21.90 \\
\hline 327 & 4.dez. 88 & 368 & 7.1 & 200.40 & 65. & 30.1 & 17.9 & 24.00 \\
\hline 387 & 4.fev. 89 & 373 & 5.6 & 298.30 & 68. & 29.9 & 19.6 & 24.70 \\
\hline 447 & 4.abr. 89 & 329 & 7.8 & 103.90 & 65. & 29.6 & 16.5 & 23.0 \\
\hline 507 & 4.jun. 89 & 232 & 6.0 & 47.00 & 71. & 24.1 & 10.5 & 17.30 \\
\hline
\end{tabular}

(*) Médias mensais

Idade em dias após a semeadura.

Fonte: DEPARTAMENTO DE FÍSICA E METEOROLOGIA DA ESALQ/USP, Piracicaba-SP. 
Tabela 3 - Acúmulo de matéria seca e altura de plantas de urucu em diferentes idades (*).

\begin{tabular}{|c|c|c|c|c|}
\hline \multirow{2}{*}{$\begin{array}{l}\text { Idade } \\
\text { (dias) }\end{array}$} & \multirow{2}{*}{$\begin{array}{c}\text { Parte } \\
\text { Analisada }\end{array}$} & \multicolumn{2}{|c|}{ Matéria Seca } & \multirow{2}{*}{$\begin{array}{l}\text { Altura } \\
(\mathrm{cm})\end{array}$} \\
\hline & & g/planta & $\mathrm{kg} / \mathrm{ha}$ & \\
\hline \multirow[t]{3}{*}{115} & foihas & 8.93 & 3.57 & - \\
\hline & ramos & 3.98 & 1.58 & - \\
\hline & total & 12.91 & 5.16 & 40.0 \\
\hline \multirow[t]{3}{*}{176} & folhas & 16.09 & 6.43 & - \\
\hline & ramos & 4.00 & 1.60 & - \\
\hline & total & 20.08 & 8.03 & 51.0 \\
\hline \multirow[t]{3}{*}{206} & folhas & 27.25 & 10.90 & - \\
\hline & ramos & 16.73 & 6.69 & - \\
\hline & total & 43.98 & 17.59 & 66.6 \\
\hline \multirow[t]{3}{*}{267} & folhas & 55.63 & 22.25 & - \\
\hline & ramos & 67.65 & 27.06 & - \\
\hline & total & 123.28 & 49.31 & 86.4 \\
\hline \multirow[t]{3}{*}{327} & folhas & 274.58 & 109.83 & - \\
\hline & ramos & 195.58 & 78.23 & - \\
\hline & total & 470.16 & 188.06 & 106.0 \\
\hline \multirow[t]{3}{*}{387} & folhas & 651.50 & 260.60 & - \\
\hline & ramos & 530.00 & 212.00 & - \\
\hline & total & 1181.50 & 472.60 & 171.8 \\
\hline \multirow[t]{4}{*}{447} & folhas & 1280.8 & 512.3 & - \\
\hline & ramos & 1467.39 & 586.96 & - \\
\hline & órg.repr. & 268.29 & 107.32 & - \\
\hline & total & 3015.66 & 1206.30 & 216.0 \\
\hline \multirow[t]{3}{*}{507} & casca & 445.90 & 178.36 & - \\
\hline & sementes & 597.33 & 238.93 & - \\
\hline & total & 1043.23 & 417.29 & 241.0 \\
\hline
\end{tabular}

(*) Média de quatro repetições por idade.

Altura de plantas medidas a partir da última gema até o solo. Idade em dias após a semeadura.

População de 400 plantas/ha' . 
Tabela 4 - Teor e extração de macronutrientes por plantas de urucu em diferentes idades (*).

\begin{tabular}{|c|c|c|c|c|c|c|c|c|c|c|}
\hline \multirow[t]{2}{*}{ Idade } & \multirow{2}{*}{$\begin{array}{l}\text { Parte } \\
\text { Analisada }\end{array}$} & \multicolumn{3}{|c|}{$\mathbf{N}$} & \multicolumn{3}{|c|}{$\mathbf{P}$} & \multicolumn{3}{|c|}{$\mathbf{K}$} \\
\hline & & $\%$ & $\mathrm{mg} /$ plant & kg/ha & $\%$ & $\mathrm{mg} /$ plant & $\mathrm{kg} / \mathrm{ha}$ & $\%$ & $\mathrm{mg} /$ plant & $\mathrm{kg} / \mathrm{ha}$ \\
\hline \multirow[t]{3}{*}{115} & folhas & 3.37 & 300.94 & 0.12 & 0.22 & 24.11 & 0.01 & 2.55 & 227.71 & 0.09 \\
\hline & $\begin{array}{l}\text { ramos }+ \\
\text { tronco }\end{array}$ & 1.60 & 63.68 & 0.03 & 0.18 & 7.16 & 0.00 & 2.45 & 97.51 & 0.04 \\
\hline & total & - & 364.62 & 0.15 & - & 31.28 & 0.01 & - & 325.23 & 0.13 \\
\hline \multirow[t]{3}{*}{176} & folhas & 3.33 & 535.46 & 0.21 & 0.33 & 53.06 & 0.02 & 1.90 & 305.52 & 0.12 \\
\hline & $\begin{array}{l}\text { ramos }+ \\
\text { tronco }\end{array}$ & 2.09 & 83.20 & 0.03 & 0.23 & 9.20 & 0.00 & 1,93 & 7.20 & 0.03 \\
\hline & total & - & 618.66 & 0.25 & - & 62.26 & 0.02 & - & 382.82 & 0.15 \\
\hline \multirow[t]{3}{*}{206} & folhas & 3.20 & 872.00 & 0.35 & 0.26 & 70.85 & 0.03 & 1.90 & 517.75 & 0.21 \\
\hline & $\begin{array}{l}\text { ramos }+ \\
\text { tronco }\end{array}$ & 1.65 & 276.04 & 0.11 & 0.23 & 36.48 & 0.02 & 1.85 & 309.50 & 0.12 \\
\hline & total & - & 1148.05 & 0.46 & - & 109.33 & 0.04 & - & 827.25 & 0.33 \\
\hline \multirow[t]{3}{*}{267} & folhas & 2.78 & 1546.51 & 0.52 & 0.20 & 111.26 & 0.04 & 1.55 & 862.26 & 0.34 \\
\hline & $\begin{array}{l}\text { ramos }+ \\
\text { ramos }\end{array}$ & 1.28 & 865.92 & 0.35 & 0.13 & 87.95 & 0.04 & 1.20 & 811.80 & 0.32 \\
\hline & total & - & 2412.43 & 0.96 & - & 199.21 & 0.08 & - & 1674.07 & 0.67 \\
\hline \multirow[t]{3}{*}{327} & folhas & 2.33 & 6397.71 & 2.56 & 0.20 & 549.16 & 0.22 & 0.98 & 2690.88 & 1.08 \\
\hline & $\begin{array}{l}\text { ramos }+ \\
\text { tronco }\end{array}$ & 0.95 & 1858.01 & 0.74 & 0.13 & 254.25 & 0.10 & 0.73 & 1427.73 & 0.57 \\
\hline & total & - & 8255.72 & 3.30 & - & 803.41 & 0.32 & - & 4118.62 & 1.65 \\
\hline \multirow[t]{3}{*}{387} & folhas & 1.95 & 12704.25 & 5.08 & 0.20 & 1303.00 & 0.52 & 1.10 & 7166.50 & 2.87 \\
\hline & $\begin{array}{l}\text { ramos }+ \\
\text { troncos }\end{array}$ & 0.75 & 3975.00 & 1.59 & 0.13 & 689.00 & 0.28 & 1.13 & 5989.00 & 2.40 \\
\hline & total & - & 16679.25 & 6.67 & - & 1992.00 & 0.80 & - & 13155.50 & 5.26 \\
\hline \multirow[t]{4}{*}{447} & folhas & 2.33 & 29825.86 & 11.93 & 0.26 & 3328.21 & 1.33 & 0.89 & 11392.71 & 4.56 \\
\hline & $\begin{array}{l}\text { ramos }+ \\
\text { tronco }\end{array}$ & 0.88 & 12913.03 & 5.17 & 0.20 & 2934.78 & 1.17 & 0.69 & 10124.99 & 4.05 \\
\hline & órg.repr. & 2.59 & 6948.71 & 2.78 & 0.40 & 1073.16 & 0.43 & 0.99 & 2656.07 & 9.67 \\
\hline & total & - & 49687.61 & 19.88 & - & 7336.15 & 2.93 & - & 24173.77 & 9.67 \\
\hline \multirow[t]{3}{*}{507} & casca & 1.03 & 4592.77 & 1.84 & 0.14 & 624.26 & 0.25 & 1.35 & 6019.65 & 2.41 \\
\hline & sementes & 1.68 & 10035.14 & 4.01 & 0.39 & 2329.59 & 0.93 & 1.95 & 11647.93 & 4.66 \\
\hline & total & - & 14627.91 & 5.85 & - & 2835.85 & 1.18 & - & 17667.58 & 7.07 \\
\hline
\end{tabular}


Tabela 4 - Continuação.

\begin{tabular}{|c|c|c|c|c|c|c|c|c|c|c|}
\hline \multirow[t]{2}{*}{ Idade } & \multirow{2}{*}{$\begin{array}{l}\text { Parte } \\
\text { Analisada }\end{array}$} & \multicolumn{3}{|c|}{$\mathrm{Ca}$} & \multicolumn{3}{|c|}{$\mathbf{M g}$} & \multicolumn{3}{|c|}{$\mathbf{s}$} \\
\hline & & $\%$ & mg/plant & kg/ha & $\%$ & mg/plant & kg/ha & $\mathscr{q}$ & mg/plant & kg/ha \\
\hline \multirow[t]{3}{*}{115} & folhas & 0.80 & 71.44 & 0.03 & 0.38 & 33.93 & 0.01 & 0.28 & 25.00 & 0.01 \\
\hline & $\underset{\text { tronco }}{\text { ramos }}+$ & 0.28 & 11.14 & 0.00 & 0.28 & 11.14 & 0.00 & 0.20 & 7,96 & 0.00 \\
\hline & total & - & 82.58 & 0.03 & - & 45.08 & 0.02 & - & 32.96 & 0.01 \\
\hline \multirow[t]{3}{*}{176} & folhas & 1.20 & 192.96 & 0.08 & 0.38 & 61.10 & 0.02 & 0.28 & 45.02 & 0.02 \\
\hline & $\underset{\text { tronco }}{\text { ramos }}+$ & 0.35 & 14.00 & 0.01 & 0.30 & 12.00 & 0.00 & 0.33 & 13.20 & 0.01 \\
\hline & total & - & 206.96 & 0.08 & - & 73.10 & 0.03 & - & 58.22 & 0.02 \\
\hline \multirow[t]{3}{*}{206} & folhas & 1.95 & 531.38 & 0.21 & 0.38 & 103.55 & 0.04 & 0.25 & 68.13 & 0.03 \\
\hline & $\underset{\text { tronco }}{\text { ramos }}+$ & 0.63 & 105.40 & 0.04 & 0.35 & 58.55 & 0.02 & 0.20 & 33.46 & 0.01 \\
\hline & total & - & 636.77 & 0.25 & - & 162.11 & 0.06 & - & 101.59 & 0.04 \\
\hline \multirow[t]{3}{*}{267} & folhas & 1.75 & 973.53 & 0.39 & 0.35 & 194.71 & 0.08 & 0.23 & 127.95 & 0.05 \\
\hline & $\begin{array}{l}\text { ramos }+ \\
\text { ramos }\end{array}$ & 0.43 & 290.89 & 0.12 & 0.20 & 135.30 & 0.05 & 0.13 & 87.95 & 0.04 \\
\hline & total & - & 1264.42 & 0.51 & - & 330.00 & 0.13 & - & 215.89 & 0.09 \\
\hline \multirow[t]{3}{*}{327} & folhas & 1.40 & 3844.12 & 1.54 & 0.40 & 1098.32 & 0.44 & 0.20 & 549.16 & 0.22 \\
\hline & $\begin{array}{l}\text { ramos + } \\
\text { tronco }\end{array}$ & 0.55 & 1075.69 & 0.43 & 0.35 & 684.53 & 0.27 & 0.13 & 254.25 & 0.10 \\
\hline & total & - & 4919.81 & 1.97 & - & 1782.85 & 0.71 & - & 803.41 & 0.32 \\
\hline \multirow[t]{3}{*}{387} & folhas & 1.38 & 8990.70 & 3.60 & 0.43 & 2801.45 & 1.12 & 0.20 & 1303.00 & 0.52 \\
\hline & $\begin{array}{l}\text { ramos }+ \\
\text { troncos }\end{array}$ & 0.28 & 1484.00 & 0.59 & 0.15 & 795.00 & 0.32 & 0.08 & 424.00 & 0.17 \\
\hline & total & - & 10474.70 & 4.19 & - & 3596.45 & 1.44 & - & 1727.00 & 0.69 \\
\hline \multirow[t]{4}{*}{447} & folhas & 1.96 & 25089.57 & 10.04 & 0.40 & 5120.32 & 2.05 & 0.15 & 1920.12 & 0.77 \\
\hline & $\underset{\text { tronco }}{\text { ramos }}+$ & 0.59 & 8657.60 & 3.46 & 0.22 & 3228.25 & 1.29 & 0.14 & 2054.35 & 0.82 \\
\hline & órg.repr. & 0.90 & 2414.61 & 0.97 & 0.34 & 912.19 & 0.36 & 0.27 & 724.38 & 0.29 \\
\hline & total & - & 36161.78 & 14.46 & - & 9260.76 & 3.70 & - & 4698.85 & 1.88 \\
\hline \multirow[t]{3}{*}{507} & casca & 0.62 & 2764.58 & 1.11 & 0.06 & 267.54 & 0.11 & 0.16 & 713.44 & 0.29 \\
\hline & sementes & 0.37 & 2210.12 & 0.88 & 0.26 & 1553.06 & 0.62 & 0.15 & 896.00 & 0.36 \\
\hline & total & - & 4974,70 & 1.99 & - & 1820.60 & 0.73 & - & 1609.43 & 0.64 \\
\hline
\end{tabular}

$\left.{ }^{*}\right)$ Média de quatro repetiçōes por idade

Cálculo da extração feito a partir da matéria seca.

Idade em dias após a semeadura.

População de 400 plantas/ha'- 
Tabela 5 - Teor e extração de macronutrientes por plantas de urucu em diferentes idades (*).

\begin{tabular}{|c|c|c|c|c|c|c|c|c|c|c|}
\hline \multirow[t]{2}{*}{ Idade } & \multirow{2}{*}{$\begin{array}{l}\text { Parte } \\
\text { Analisada }\end{array}$} & \multicolumn{3}{|c|}{$\mathbf{B}$} & \multicolumn{3}{|c|}{$\mathrm{Cu}$} & \multicolumn{3}{|c|}{$\mathrm{Fe}$} \\
\hline & & ppm & eg/plant & $\mathrm{g} / \mathrm{ha}$ & ppm & $\mu \mathrm{g} /$ plant & g/ha & $\mathrm{ppm}$ & eg/plant & gha \\
\hline \multirow[t]{3}{*}{115} & folhas & 55.00 & 491.15 & 0.20 & 8.25 & 73.67 & 0.03 & 245.00 & 2187.85 & 0.88 \\
\hline & $\underset{\text { tronco }}{\text { ramos }}+$ & 40.75 & 162.19 & 0.06 & 9.75 & 38.80 & 0.02 & 67.75 & 269.64 & 0.11 \\
\hline & total & 50.61 & 653.34 & 0.26 & 6.71 & 112.48 & 0.04 & 190.36 & 2457.49 & 0.98 \\
\hline \multirow[t]{3}{*}{176} & folhas & 44.75 & 719.58 & 0.29 & 13.75 & 221.10 & 0.09 & 274.50 & 4413.96 & 1.77 \\
\hline & $\begin{array}{l}\text { ramos }+ \\
\text { tronco }\end{array}$ & 39.50 & 158.00 & 0.06 & 13.25 & 53.00 & 0.02 & 82.75 & 331.00 & 0.13 \\
\hline & total & 43.70 & 877.58 & 0.35 & 13.65 & 274.10 & 0.11 & 236.30 & 4744.96 & 1.90 \\
\hline \multirow[t]{3}{*}{206} & folhas & 41.50 & 1130.88 & 0.45 & 21.50 & 585.88 & 0.23 & 495.75 & 13509.19 & 5.40 \\
\hline & $\begin{array}{l}\text { ramos }+ \\
\text { tronco }\end{array}$ & 34.00 & 568.82 & 0.23 & 20.00 & 334.60 & 0.13 & 160.00 & 2676.80 & 1.07 \\
\hline & total & 38.65 & 1699.70 & 0.68 & 20.93 & 920.48 & 0.37 & 368.03 & 16185.99 & 6.47 \\
\hline \multirow[t]{3}{*}{267} & folhas & 33.50 & 1863.61 & 0.75 & 5.50 & 305.97 & 0.12 & 367.75 & 20457.93 & 8.18 \\
\hline & $\begin{array}{l}\text { ramos }+ \\
\text { ramos }\end{array}$ & 23.25 & 1572.86 & 0.63 & 5.00 & 338.25 & 0.14 & 77.25 & 5225.96 & 2.09 \\
\hline & total & 27.88 & 3436.47 & 1.37 & 5.23 & 644.22 & 0.26 & 208.34 & 25683.90 & 10.27 \\
\hline \multirow[t]{3}{*}{327} & folhas & 31.00 & 8511.98 & 3.40 & 4.50 & 1235.61 & 0.49 & 180.75 & 49630.33 & 19.85 \\
\hline & $\begin{array}{l}\text { ramos }+ \\
\text { tronco }\end{array}$ & 26.50 & 5182.87 & 2.07 & 4.25 & 831.22 & 0.33 & 47.00 & 9192.26 & 3.68 \\
\hline & total & 29.13 & 13694.85 & 5.48 & 4.40 & 2066.82 & 0.83 & 125.11 & 58822.60 & 23.53 \\
\hline \multirow[t]{3}{*}{387} & folhas & 44.00 & 28666.00 & 11.47 & 10.75 & 7003.63 & 2.80 & 247.75 & 161409.13 & 64.56 \\
\hline & $\begin{array}{l}\text { ramos }+ \\
\text { troncos }\end{array}$ & 22.00 & 11660.00 & 4.66 & 9.25 & 4902.50 & 1.96 & 62.00 & 32860.00 & 13.14 \\
\hline & total & 34.13 & 40326.00 & 16.13 & 10.08 & 11906.13 & 4.76 & 164.43 & 194269.13 & 77.71 \\
\hline \multirow[t]{4}{*}{447} & folhas & 43.50 & 55683.48 & 22.27 & 7.50 & 9600.60 & 3.84 & 201.00 & 257296.08 & 102.92 \\
\hline & $\begin{array}{l}\text { ramos }+ \\
\text { tronco }\end{array}$ & 31.13 & 45679.85 & 18.27 & 66.00 & 8804.34 & 3.52 & 43.50 & 63831.47 & 25.53 \\
\hline & org.repr. & 50.00 & 13414.50 & 5.37 & 13.25 & 3554.84 & 1.42 & 104.76 & 28106.06 & 11.24 \\
\hline & total & 38.06 & 114777.83 & 45.91 & 7.28 & 21959.78 & 8.78 & 115.80 & 349233.61 & 139.69 \\
\hline \multirow[t]{3}{*}{507} & casca & 18.75 & 8360.63 & 3.34 & 16.00 & 7134.40 & 2.85 & 439.00 & 195750.10 & 78.30 \\
\hline & sementes & 30.00 & 17919.90 & 7.17 & 19.13 & 11426.92 & 4.57 & 92.75 & 55402.36 & 22.16 \\
\hline & total & 25.19 & 26280.53 & 10.51 & 17.79 & 18561.32 & 7.42 & 240.75 & 251152.46 & 100.46 \\
\hline
\end{tabular}

(*) Média de quatro repetições por idade

Cálculo da extração feito a partir da matéria seca.

Idade em dias após a semeadura.

População de 400 plantas/ha-1. 
- Os limites das concentrações dos micronutrientes nas folhas $\epsilon$ de: $\mathrm{B}-33,5 \mathrm{ppm}-55,0 \mathrm{ppm}$; $\mathrm{Cu}-4,5 p p m-21,5 p p m ; \mathrm{Fe}$ - 180,7ppm-495,7ppm; Mn - 71,0ppm-108,5ppm; Zn - 30,7ppm-57,7ppm.

- A extração dos macro e micronutrientes por uma cultura de 440 plantas/ha aos 507 dias $\varepsilon$ de: $N$ 25,7kg; P - 4,1kg; $\mathrm{K}-16,7 \mathrm{~kg} ; \mathrm{Ca}-16,45 \mathrm{~kg} ; \mathrm{Mg}-$ 4,4kg; S - 2,5kg; B - 56,4g; Cu - 16,2g; Fe - 240,1g; $\mathrm{Mn}-130,3 \mathrm{~g}$ e $\mathrm{Zn}-50,7 \mathrm{~g}$.

\section{REFERENCIAS BIBLIOGRAFICAS}

ANUÁRIO ESTATÍSTICO DO BRASIL - 1990, Rio de Janeiro, IBGE, 1990.

BALIANE, A. Cultura do urucueiro. EMATER-RJ. 10p., 1982.

CARVALHO, E.R. Bibliografia do urucu (Bixa orellana). EMBRAPA/CENARGE, Brasília, DF. 59p., 1986.

HAAG, H.P.; DECHEN, A.R.; ROSOLEN, D.L. Carência de macronutrientes e de boro em plantas de urucu. Anais da Escola Superior de Agricultura "Luiz de Queiroz", Piracicaba, 45(2): 423-31, 1988.

JOLY, A.B. Botânica. Introdução daxonomia vegetal. São Paulo,SP, Companhia Editora Nacional, 1977.

ROSALEN, D.L.; SIMÃo, S.; HAAG, H.P. Considerações sobre a cultura do urucu (Bixa orellana L.). Informativo Técnico, Piracicaba (12): 1-41, 1991.

SARRUGE, J.R.; HAAG, H.P. Análise química em plantas. Piracicaba, ESALQ, Departamento de Química, 1974. 56p.

Trabalho entregue para publicação em 22.03.91

Trabalho aprovado para publicação em 13.08.91 